\title{
Impacts of Hysteresis and Magnetic Couplings on the Stability Domain of Ferroresonance in Asymmetric Three-Phase Three-Leg Transformers
}

\author{
Paul S. Moses, Student Member, IEEE, Mohammad A. S. Masoum, Senior Member, IEEE, \\ and Hamid A. Toliyat, Fellow, IEEE
}

\begin{abstract}
This paper investigates the stability domain of ferroresonance in asymmetric three-phase three-leg transformers considering magnetic couplings and hysteresis effects of the core. A newly developed and accurate time-domain transformer model capable of simulating dynamic and transient operating conditions is implemented in this study. The model is based on electromagnetic circuit theory and considers dynamic hysteresis effects (major and minor loops) as well as core topology, asymmetry, and magnetic flux cross-coupling interactions of the core legs. Unbalanced switching with series and shunt capacitances, which is known to increase the risk of ferroresonance, is studied with the developed model. The validity of the model under ferroresonant conditions is confirmed by comparisons with extensive experimental data. The main contribution is a new analysis of (a)symmetric three-phase transformer ferroresonance behavior with an accurate core model capable of predicting ferroresonance modes.
\end{abstract}

Index Terms-Ferroresonance, hysteresis, nonlinear and threephase transformer model.

\section{INTRODUCTION}

$\mathbf{M}$ ANY investigations of ferroresonance phenomena in power and instrument transformers have been performed, spanning nearly a century of accumulated research [1], [2]. Much progress has been made in the modeling and understanding of single-phase transformer ferroresonance. However, one of the weakest areas in ferroresonance research remains in the modeling of (a)symmetric three-phase transformers.

\section{A. Description of Ferroresonance Problem}

Ferroresonance is a complex oscillatory interaction of energy exchanged between nonlinear magnetizing inductances of ferromagnetic cores and system capacitances (e.g., series compensated lines) [1], [3]. These oscillations manifest as large distorted voltages and currents potentially leading to excessive heating and insulation failure in transformers as well as significant disruptions to power networks [4]-[11].

Manuscript received May 19, 2010; revised August 23, 2010; accepted October 4, 2010. Date of publication December 17, 2010; date of current version May 18, 2011. Paper no. TEC-00219-2010.

P. S. Moses and M. A. S. Masoum are with the Department of Electrical and Computer Engineering, Curtin University, Perth, WA 6845, Australia (e-mail: paul.s.moses@gmail.com; m.masoum@curtin.edu.au).

H. A. Toliyat is with the Department of Electrical and Computer Engineering, Texas A\&M University, College Station, TX 77843 USA (e-mail: toliyat@ece.tamu.edu).

Color versions of one or more of the figures in this paper are available online at http://ieeexplore.ieee.org.

Digital Object Identifier 10.1109/TEC.2010.2088400
Unlike ordinary $R L C$ circuit resonance, oscillations involving nonlinear magnetizing inductances can exhibit multiple modes with no definite resonant frequency. These modes typically come in four types. The fundamental mode oscillates at the system frequency $f$ and usually contains harmonics. The subharmonic mode is periodic and oscillates at $1 / n$ multiples of the system frequency $(f / n)$. Quasi-periodic modes exhibit irregular patterns of periodicity due to incommensurable frequencies $n f_{1}+m f_{2}$, where $f_{1} / f_{2}$ is irrational with integer $n$ and $m$. These modes are strictly nonperiodic and produce a discontinuous frequency spectrum. Chaotic modes exhibit no periodicity and produce a continuous frequency spectrum resembling broadband noise. Sudden jumps (bifurcations) to different modes are also possible due to gradual system variations and perturbations (e.g., switching transients) [3].

\section{B. Historical Review of Ferroresonance Research}

Ferroresonance research has its origins in ordinary transformer resonance studies performed as early as 1907 [12]. It was not until 1920 that the vernacular "ferroresonance" was first documented by Boucherot [13] describing the unusual coexisting operating points in a series resistor, nonlinear inductor and capacitor circuit. Much early experience in ferroresonance was gained through extensive field observations when digital computer models and analytical methods were in their infancy. Notable examples are the studies conducted in [14]-[17] on distribution systems exhibiting ferroresonance with voltage regulation capacitors, and the triggering of ferroresonance in threephase systems from single-pole switching.

Analytical approaches based on simple graphical solutions were proposed early on [18], [19] to predict particular bifurcations in single-phase ferroresonant circuits. It was not until the late 1980s that significant breakthroughs were made in nonlinear transformer models and analytical techniques. This was largely driven by advancements in computing power and the foundation of nonlinear dynamics and chaos theory, which Kieny [20] and Mork and Stuehm [21] first proposed as a suitable framework for ferroresonance study. Henceforth, new useful analytical approaches have emerged (e.g., bifurcation, phase-plane and Poincaré techniques [22]-[24]) which are now the benchmark for modern ferroresonance analyses.

Toward the end of the 20th century, the study area has branched into four main directions: 1) practical system level case studies [6], [25], [26]; 2) ferroresonance identification methods [27], [28]; 3) development of ferroresonance mitigation 
approaches [29]-[33]; and most prominently, 4) the improvement of analytical techniques and the modeling of electromagnetic transients in transformers, which is the main contribution of this paper.

\section{Modeling of Three-Phase Ferroresonance Phenomena}

There have been very few attempts at studying three-phase transformers under ferroresonance conditions. The work given in [34]-[36] implement models considering core topology and employ single-value nonlinear functions for core effects. Analytical and numerical approaches are developed in [37]-[39] to calculate ferroresonance modes. However, these approaches do not consider dynamic hysteresis effects with core asymmetry and associated magnetic leg couplings. Furthermore, these models lack experimental validation.

\section{New Ferroresonance Studies With Hysteretic Core Models}

A new class of transformer models is emerging with the inclusion of hysteresis nonlinearities into core representations. Recent studies have shown that accurate representation of ferromagnetic iron-core nonlinearities (e.g., saturation, hysteresis, and eddy currents) is important in ferroresonance. Hysteresis formation significantly impacts the stability domain of ferroresonance, especially for subharmonic and chaotic modes in singlephase transformer core models [40], [41]. The common approach of approximating core nonlinearities with nonhysteretic single-value functions (e.g., piece-wise, exponential and polynomials) has proven to be inadequate for ferroresonance studies in single-phase transformers. The impacts of dynamic hysteresis nonlinearities (e.g., major and minor loops) and magnetically coupled asymmetric legs on three-phase transformer ferroresonance have not been explored.

For the first time, this paper studies ferroresonance in asymmetric three-phase transformers using a newly developed and detailed nonlinear electromagnetic circuit model for multi-leg transformer cores. The model is implemented in time domain and considers core asymmetry and topology, magnetic couplings of the legs, as well as nonlinearities in the iron-core structure. Accurate dynamic hysteresis nonlinearities including major and minor loop effects are implemented for each magnetically coupled core-leg. Experimental validation tests have been performed to demonstrate the models high accuracy in duplicating ferroresonance behavior in three-phase transformers.

\section{ThreE-PhASE TRANSFORMER MODELING APPROACH}

There has been significant progress in the understanding and modeling of single-phase transformer ferroresonance [23], [42]-[45]. However, exact behavior of three-phase transformers under ferroresonance conditions is largely unexplored due to the lack of adequate core models. These models present significant challenges due to the magnetic circuits of different core topologies (e.g., three-leg, five-leg) and associated magnetic couplings in the iron-core structure. Moreover, a three-phase transformer core model cannot be simplified to three single-phase transformers. Each branch of the three- phase core structure has unique

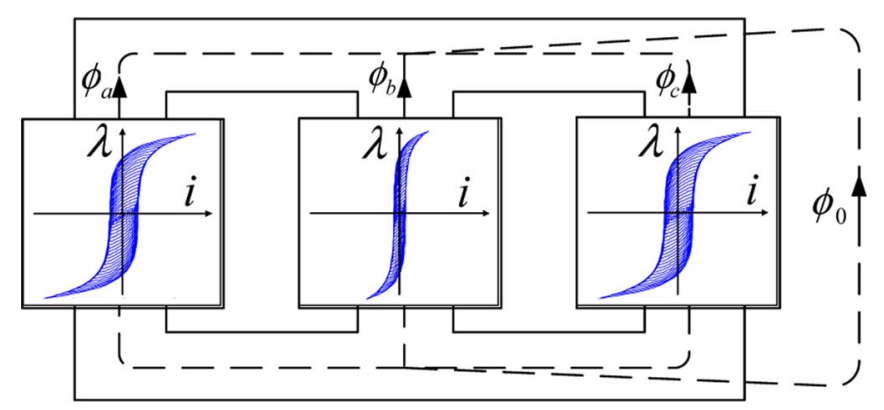

Fig. 1. Asymmetric three-phase three-leg transformer core.

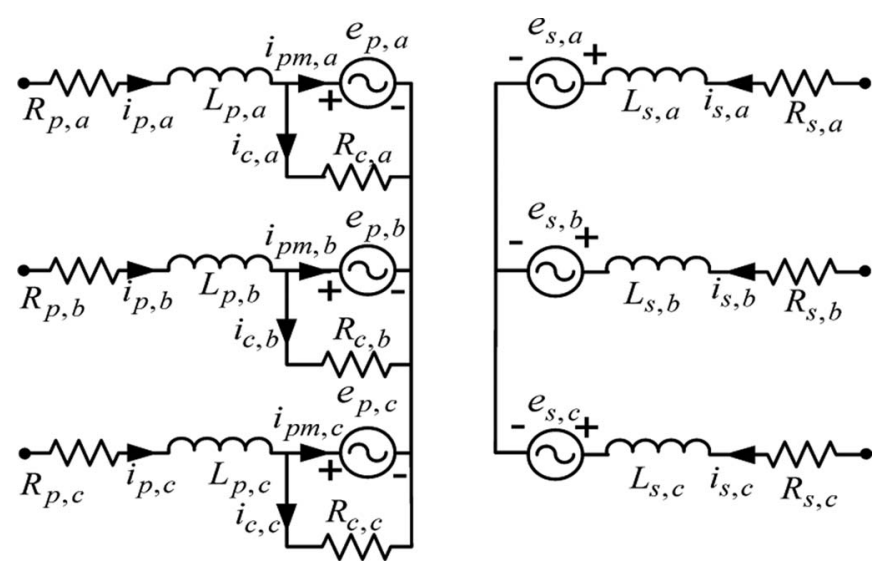

Fig. 2. Three-phase transformer electric equivalent circuit (wye/wye).

nonlinear leg and yoke reluctance characteristics due to varying core geometries and flux path lengths. This is referred to as an asymmetric three-phase transformer which has not been studied under ferroresonance conditions.

In this paper, a newly developed three-phase transformer model [46] is implemented and its performance under ferroresonance conditions is examined. The most significant feature of the developed model is the inclusion of detailed hysteresis core models considering major and minor loop effects combined with an asymmetric three-leg electromagnetic structure (see Fig. 1).

\section{A. Transformer Electromagnetic Circuits}

The duality principle is applied in the development of coupled electric and magnetic equivalent circuit models where fluxes are computed as currents, magnetomotive forces (MMFs) as voltages and reluctances as resistances [2], [47]-[49]. The electric circuit represents the ohmic losses in the windings as well as leakage flux inductances and eddy-current core losses. The electric couplings in the phases due to winding connections (e.g., wye, delta) are accounted for in the electric circuit (see Fig. 2).

The magnetic circuit is necessary for modeling the different core topologies, multiple flux paths and corresponding couplings in the magnetic legs (see Fig. 3). Each magnetic leg uses an expanded nonlinear core model to represent dynamic hysteresis nonlinearities in each leg. In this way, the hysteresis functions in each core leg can be individually adjusted to represent core asymmetry in the magnetic circuit. The governing electric and magnetic circuit relationships based on Kirchoff's 


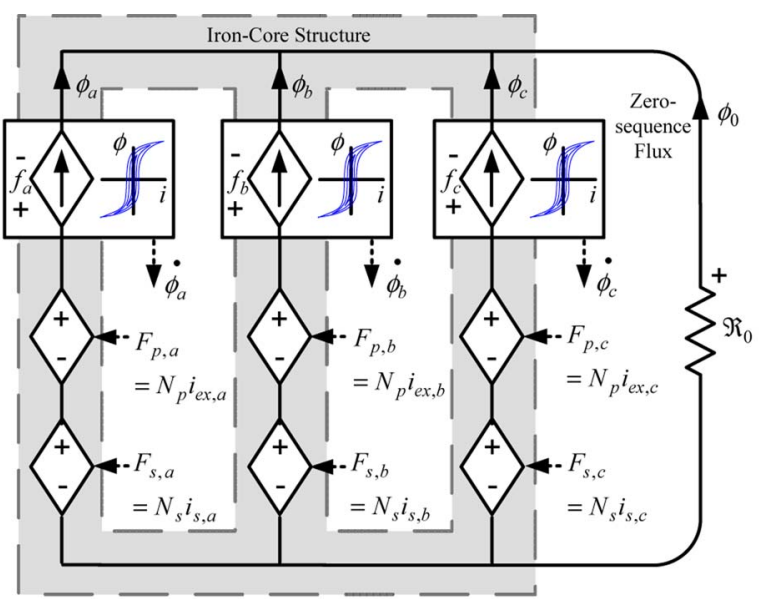

(a)

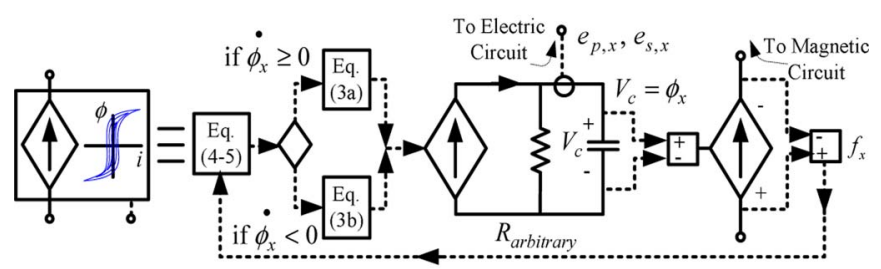

(b)

Fig. 3 (a) Three-phase three-leg transformer magnetic equivalent circuit and (b) expanded view of hysteresis subcircuit for modeling each core leg.

voltage and current laws are

$$
\begin{gathered}
v_{p, x}(t)=R_{p, x} i_{p, x}(t)+L_{p, x} \frac{d i_{p, x}(t)}{d t}+\frac{d \lambda_{p, x}(t)}{d t} \\
v_{s, x}(t)=R_{s, x} i_{s, x}(t)+L_{s, x} \frac{d i_{s, x}(t)}{d t}+\frac{d \lambda_{s, x}(t)}{d t} \\
(x=a, b, c) \\
\phi_{a}+\phi_{b}+\phi_{c}+\phi_{0}=0
\end{gathered}
$$

where the respective primary/secondary terminal voltages, winding currents, and flux linkages for each phase $x$ are $v_{p, x}$, $v_{s, x}, i_{p, x}, i_{s, x}, \lambda_{p, x}$, and $\lambda_{s, x}$. The primary/secondary phase winding resistances and inductances are $R_{p, x}, R_{s, x}, L_{p, x}$, and $L_{s, x}$, respectively. The magnetic circuit fluxes in each leg are $\phi_{a}, \phi_{b}$, and $\phi_{c}$ with zero-sequence leakage flux $\phi_{0}$.

\section{B. Magnetic Hysteresis Core Models}

The ferroresonance phenomenon is very much dependent on the complex magnetization processes of ferromagnetic iron-core structures. Therefore, accurate core models for true hysteresis behavior including major and minor loop effects are crucial for reliable ferroresonance predictions. The approach adopted in this paper is to modify the scalar hysteresis model of [50] and integrate it into the three-leg magnetic circuit.

In order to integrate the model into the electromagnetic circuit, the original hysteresis functions [50] have been modified to compute magnetic flux and MMFs instead of $B$ and $H$

$$
\begin{aligned}
& \text { (e.g., } B \rightarrow \phi \text { and } H \rightarrow f \text { ) } \\
& \begin{array}{c}
\frac{d \phi_{x}}{d t}=\frac{d f_{x}}{d t}\left[\rho_{x}+\frac{\phi_{x}^{-}\left(f_{x}\right)-\phi_{x}}{\phi_{x}^{-}\left(f_{x}\right)-\phi_{x}^{+}\left(f_{x}\right)}\left(\frac{d \phi_{x}^{+}\left(f_{x}\right)}{d f_{x}}-\rho_{x}\right)\right] \\
\text { if } \frac{d \phi_{x}}{d t} \geq 0 \\
\frac{d \phi_{x}}{d t}=\frac{d f_{x}}{d t}\left[\rho_{x}+\frac{\phi_{x}-\phi_{x}^{+}\left(f_{x}\right)}{\phi_{x}^{-}\left(f_{x}\right)-\phi_{x}^{+}\left(f_{x}\right)}\left(\frac{d \phi_{x}^{-}\left(f_{x}\right)}{d f_{x}}-\rho_{x}\right)\right] \\
\quad \text { if } \frac{d \phi_{x}}{d t}<0 \quad(x=a, b, c) .
\end{array}
\end{aligned}
$$

The major hysteresis loop limiting ascending and descending curve functions, and instantaneous leg fluxes for each phase $x$ are $\phi_{x}^{+}\left(f_{x}\right), \phi_{x}^{-}\left(f_{x}\right)$, and $\phi_{x}$, respectively, where the leg MMF potential is $f_{x}$. The slope of the fully saturated region along the limiting hysteresis curves is approximated by $\rho_{x}$.

In order to compute the hysteresis response through (3), the ascending and descending limiting curves $\left(\phi_{x}^{+}\left(f_{x}\right), \phi_{x}^{-}\left(f_{x}\right)\right)$ must be determined for the major hysteresis loops in each core leg. The following nonlinear function is used for this purpose:

$$
\phi(f)=\operatorname{sgn}(f) \cdot \alpha \log _{e}(\beta|f|+1)
$$

where $\alpha_{x}$ and $\beta_{x}$ influence the vertical and horizontal scaling of $\phi(f)$, respectively. If (4) is transposed to the right (or left) by $\sigma_{x}$, the limiting ascending (or descending) curve functions defined for the major hysteresis loop are as follows:

$$
\begin{gathered}
\phi_{x}^{+}\left(f_{x}\right)=\operatorname{sgn}\left(f_{x}-\sigma_{x}\right) \cdot \alpha_{x} \log _{e}\left(\beta_{x}\left|f_{x}-\sigma_{x}\right|+1\right) \\
\phi_{x}^{-}\left(f_{x}\right)=\operatorname{sgn}\left(f_{x}+\sigma_{x}\right) \cdot \alpha_{x} \log _{e}\left(\beta_{x}\left|f_{x}+\sigma_{x}\right|+1\right) \\
(x=a, b, c)
\end{gathered}
$$

Therefore, $\sigma_{x}$ allows the width of the major hysteresis loop to be specified. Furthermore, by separately defining $\phi_{x}^{+}\left(f_{x}\right)$ and $\phi_{x}^{-}\left(f_{x}\right)$, nonsymmetrical hysteresis loops can be defined. The advantage of these functions is that only two parameters $\left(\alpha_{x}, \beta_{x}\right)$ are required to fit the curve to measured data. However, the curve fitting process must be carefully performed for individual core legs because the strong magnetic leg couplings can increase sensitivity to fitting errors.

The required slope functions for (3) are obtained through differentiation of (5) with respect to $\operatorname{MMF}\left(f_{x}\right)$

$$
\frac{d \phi_{x}^{ \pm}\left(f_{x}\right)}{d f_{x}}=\frac{\alpha_{x} \beta_{x}}{\beta_{x}\left|f_{x} \mp \sigma_{x}\right|+1} \quad(x=a, b, c) .
$$

In this paper, the limiting functions $\phi_{x}^{+}\left(f_{x}\right)$ and $\phi_{x}^{-}\left(f_{x}\right)$ were curve fitted to measured major hysteresis loops for each core leg obtained from the test procedures of [51] (see the Appendix for function parameters).

The modified hysteresis equations (3)-(6) estimate minor hysteresis loops based on domain wall motion theory of ferromagnetic material. The model assumes domain wall motion densities (Barkhausen jumps) vary proportionally to the growth or recession of domain regions corresponding to changes in field strength [50]. It is therefore important to note that even though the major hysteresis loop curve functions are estimated from sinusoidal excitation measurements, the model is capable of accurately estimating the magnetic response for arbitrary 


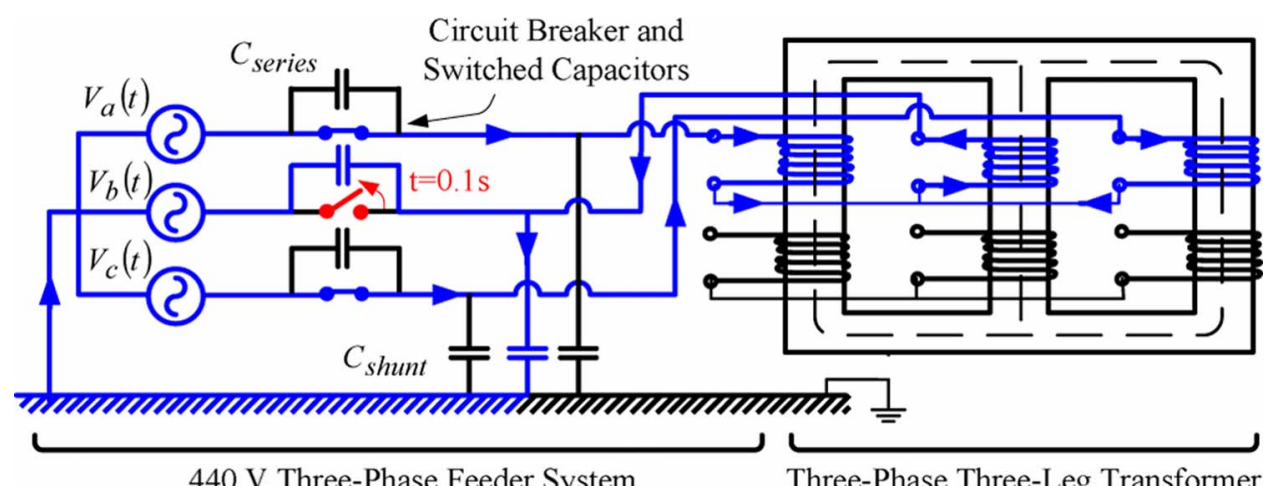

Fig. 4. Ferroresonance in an unloaded transformer fed through series and shunt capacitors (e.g., cable capacitance). The blue lines indicate the ferroresonance path when phase $b$ circuit breaker is opened (red).

nonsinusoidal excitations (e.g., ferroresonance) provided they fall within the domain of the major hysteresis loop. This is because the hysteresis equations (3) are based on the variation of excitation to compute the variation of flux for estimating minor loop formations.

Finally, the formulated hysteresis functions are realized with current sources in the electromagnetic circuit controlled by (3) and solved in time domain in PSPICE using the NewtonRaphson numerical approach (see Fig. 3). At every time step, an "IF statement" selects one of the equations (3) based on the sign of the induced voltage or flux derivative, e.g., whether magnetization is increasing or decreasing. The model parameters and transformer data are included in the Appendix.

\section{Ferroresonance System CASE Study}

The ferroresonance study for this paper is performed for an unloaded wye/wye three-phase three-leg transformer supplied by a three-phase source (see Fig. 4). The transformer primary and secondary windings are rated at 440 and $55 \mathrm{~V}(10 \mathrm{~A})$, respectively, and operate at a nominal frequency of $50 \mathrm{~Hz}$. The ferroresonance circuit consists of series and shunt capacitances interacting with the magnetizing inductances of the wound transformer core legs. The origin of $C_{\text {shunt }}$ and $C_{\text {series }}$ can typically be from circuit breakers equipped with grading capacitors, shunt and series transmission line capacitances (overhead and underground cables), reactive power compensation capacitor banks, and lumped stray capacitances in transformer windings, bushings, bus bars, and feeders.

In order for ferroresonance to occur, a system perturbation (e.g., switching transient) with initial conditions conducive to ferroresonance is usually necessary. For the studied system, phase $b$ circuit breaker is used to impose switching transients to initiate ferroresonance. There have been many such practical occurrences where unsynchronized three-phase switching or single-phase circuit breaker operations resulted in one or two phases suddenly lost while the transformer is unloaded or lightly loaded, giving rise to ferroresonance [3]. For example, feeders employing single-phase circuit breakers or fuses can suddenly develop faults causing one of the phases to deenergize. The switching transient and resulting unbalanced excitation of the transformer can lead to a ferroresonance path involving magne- tizing inductances in series with capacitances. Furthermore, in unloaded or lightly loaded systems (e.g., rural distribution feeders), there may be insufficient damping for ferroresonance. The resulting large distorted currents and over-voltages can cause severe damage to networks.

\section{Simulation Results}

Detailed time-domain simulation analysis based on the developed three-phase three-leg transformer model is carried out for the system described in Section III. Simulations are performed in PSPICE using the Newton-Raphson numerical algorithm with a variable time step limited to $50 \mu \mathrm{s}$.

At the start of each simulation run, the voltage is ramped linearly to its rated value to reach steady-state conditions and to avoid transformer inrush effects. Ferroresonance considering different combinations of $C_{\text {series }}$ and $C_{\text {shunt }}$ parameters (see Fig. 4) with single-phase switching actions are tested. The initial conditions of the system must be considered as they highly influence ferroresonance behavior. Unless otherwise stated, the reported simulation results maintain the same initial values (e.g., point-on-wave of ac voltage when the circuit breaker is opened, residual fluxes and capacitance charge). At $t=0 \mathrm{~s}$, the simulated core is assumed to have zero residual flux and all capacitors have no initial charge. The initial phase angle between the switch opening instant with respect to the phase $a$ voltage peak is stated in degrees as $\psi$.

For selected cases, time-domain waveforms of primary voltages and currents including core leg fluxes are computed. Furthermore, bifurcation diagrams, Poincaré maps, phase-plane trajectories, and hysteresis formations are plotted to better analyze the complicated stability domain of ferroresonance modes. In Section V, experimental tests are performed to verify simulated results and model accuracy.

\section{A. Bifurcation Analysis}

A useful and effective approach for identifying system parameters conducive to ferroresonance is carried out through bifurcation analysis. The shunt capacitance is chosen as the bifurcation parameter and the primary phase voltages are studied. Tests are repeated for $C_{\text {shunt }}$ ranging from 0.1 to $10 \mu \mathrm{F}$ under 


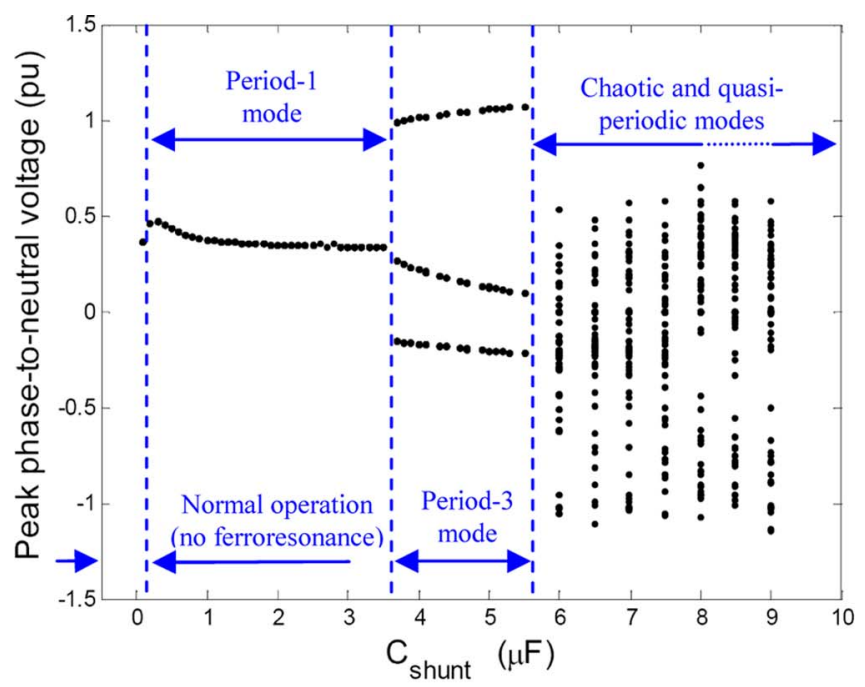

(a)

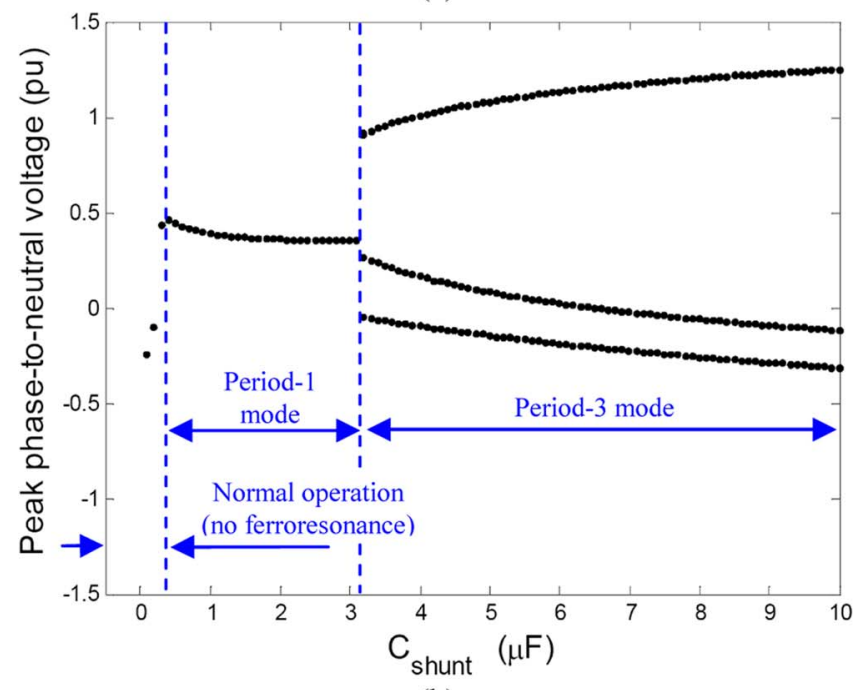

(b)

Fig. 5. Bifurcation diagram of ferroresonance modes (phase $b$ voltage) for fixed $C_{\text {series }}(=30 \mathrm{pF})$ and different $C_{\text {shunt }}$ values. (a) Considering hysteresis using the proposed three-phase three-leg transformer model and (b) without hysteresis using single-value magnetizing curves. Note the absence of chaotic ferroresonance modes and the generation of false period- 3 modes in the lower diagram when using single-value (nonhysteretic) core models.

the same single-phase fault clearing condition. The bifurcation diagram is constructed from each $C_{\text {shunt }}$ parameter change by sampling at the power system frequency $(50 \mathrm{~Hz})$ the primary voltage for the open phase $b$. The transient period is ignored in the sampling process to only analyze the subsequent stable ferroresonance oscillations. Poincaré maps are constructed in a similar way by sampling the phase-plane trajectory orbits. The resulting patterns can be interpreted for visual classification of ferroresonance modes [22].

Fig. 5 illustrates the existence of multiple ferroresonance modes in the bifurcation diagram based on the developed hysteresis core model. For $C_{\text {shunt }}=0.1$ to $0.5 \mu \mathrm{F}$, there is a small jump (bifurcation) in the operating point from normal conditions to fundamental ferroresonance mode. Fundamental ferroresonance mode is shown in Fig. 6 for $C_{\text {shunt }}=200$ pF. The circuit breaker transient is indicated by the large orbit excursions which eventually settle to a stable attracting limit cycle. The distorted noncircular orbit indicates the presence of harmonics in the voltage waveforms.

For $C_{\text {shunt }}=3.6 \mu \mathrm{F}$, another bifurcation occurs where the transformer enters period-3 type ferroresonance mode as indicated by the branching into a three line trajectory (see Fig. 5). This mode is stable up to $C_{\text {shunt }}=5.5 \mu \mathrm{F}$. Simulated timedomain waveforms and hysteresis formation for this mode are shown for $C_{\text {shunt }}=4 \mu \mathrm{F}$ in Fig. 7. The voltages, fluxes and currents exhibit highly distorted waveforms reaching in excess of $1.2,2$, and 0.4 pu of rated peak values, respectively. The phase-plane trajectory of this mode indicates multiple competing solutions (known as attractors) which influence the trajectory orbits to form subharmonic oscillations.

Beyond $C_{\text {shunt }}=5.5 \mu \mathrm{F}$, a bifurcation from period-3 into chaotic ferroresonance modes is detected. This is indicated by the nonrepeating structure of the bifurcation diagram corresponding to the sampling of chaotic voltage waveforms. This behavior is due to the existence of a strange attractor in the stability domain.

\section{B. Sensitivity of Ferroresonance to Initial Conditions}

The previous simulation studies have assumed fixed circuit parameters for a varying $C_{\text {shunt }}$. To illustrate the sensitivity of ferroresonance to initial conditions, the circuit breaker opening instant for the previous case (see Fig. 7) is now changed to $\psi=60^{\circ}$. The resulting waveforms and phase-plane trajectories in Fig. 8 now indicate a momentary ferroresonant oscillation which loses its stability and dampens out.

\section{Impact of Hysteresis on Three-Phase Three-Leg Transformer Ferroresonance}

The bifurcation diagram of Fig. 5(b) demonstrates the estimated ferroresonance modes based on a nonhysteretic core model. A single-value nonlinear saturation curve based on (4) with zero hysteresis width in each core leg is implemented for the previous case study. The predicted stability domain of ferroresonance is significantly different when compared to Fig. 5(a). The bifurcation transition into period-3 ferroresonance occurs at a lower $C_{\text {shunt }}$ value $(3.1 \mu \mathrm{F})$ without hysteresis compared to $C_{\text {shunt }}=3.6 \mu \mathrm{F}$ with hysteresis, and extends for a wider range of capacitance. More importantly, when hysteresis is neglected, the model does not detect the chaotic modes and falsely replaces them with extended period-3 subharmonic modes. These findings are consistent with the single-phase transformer study performed in [41] in which chaotic modes were also misrepresented in nonhysteretic core models. The bifurcation diagram of Fig. 5(a) has been confirmed through extensive measurements.

\section{Impact of Magnetic Couplings on Three-Phase Three-Leg Transformer Ferroresonance}

In order to assess the impact of magnetic leg couplings on three-phase transformer ferroresonance, a transformer bank (i.e., three single-phase transformers) was simulated under the 

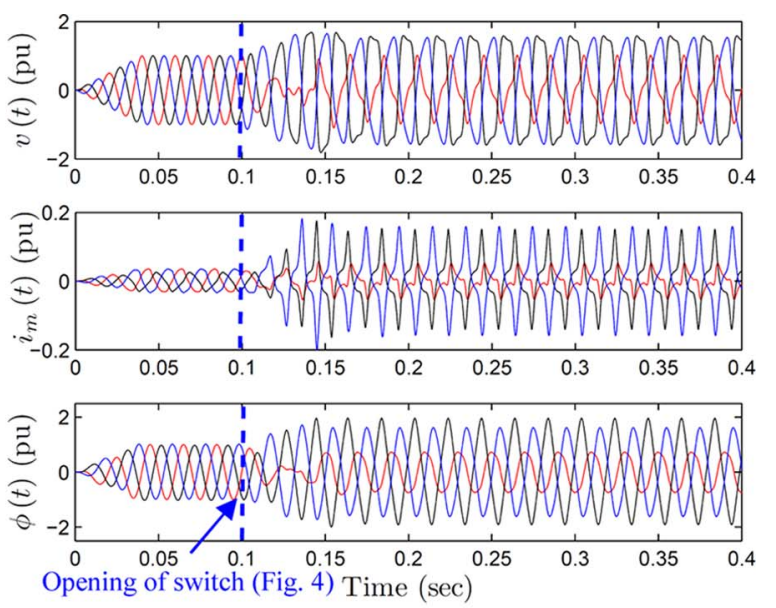

(a)

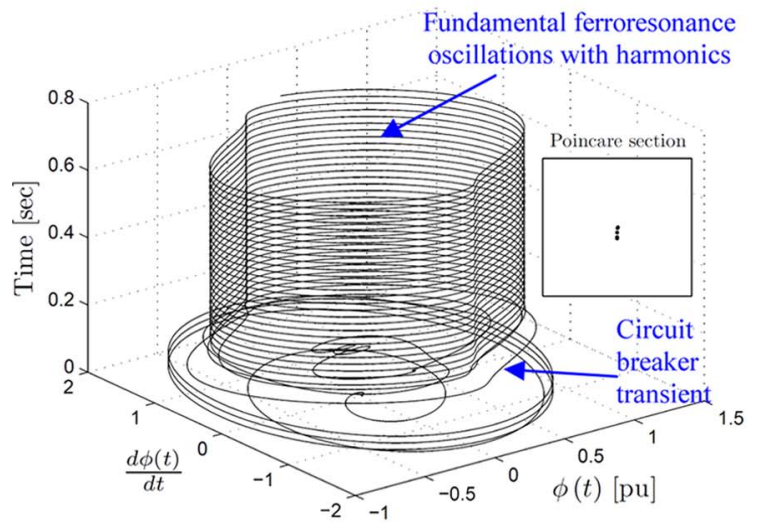

(b)

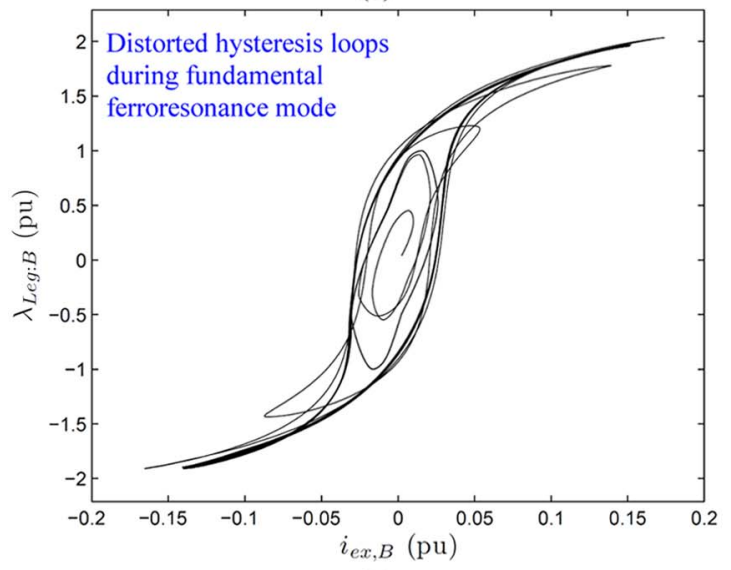

(c)

Fig. 6. Time-domain waveforms of transformer primary voltages, winding currents and core fluxes for fundamental ferroresonance mode $\left(C_{\text {series }}=\right.$ $200 \mathrm{nF}, C_{\text {shunt }}=200 \mathrm{pF}$ ). (b) Phase-plane trajectory and (c) hysteresis loop (phase $b$ ) shows the circuit breaker transient perturbing the system oscillations which then settle to a stable attracting limit cycle. The circuit breaker is opened at an initial phase angle of $\psi=90^{\circ}$ w.r.t. peak phase $a$ voltage.

same conditions described in Section III (see Fig. 4). This type of transformer has no magnetic core leg couplings because the magnetic cores of each phase are physically isolated. The developed model can approximate this behavior by setting the zero-sequence reluctance to a very small value, effectively elim-
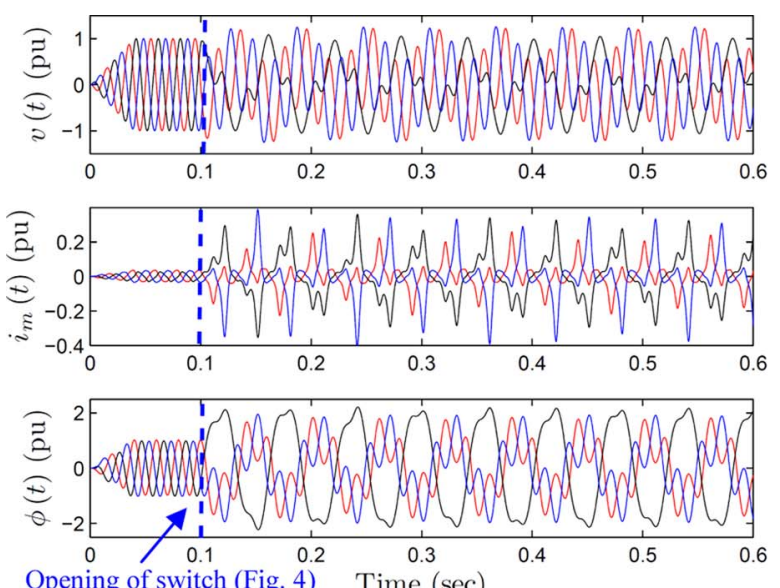

Opening of switch (Fig. 4) Time (sec)

(a)

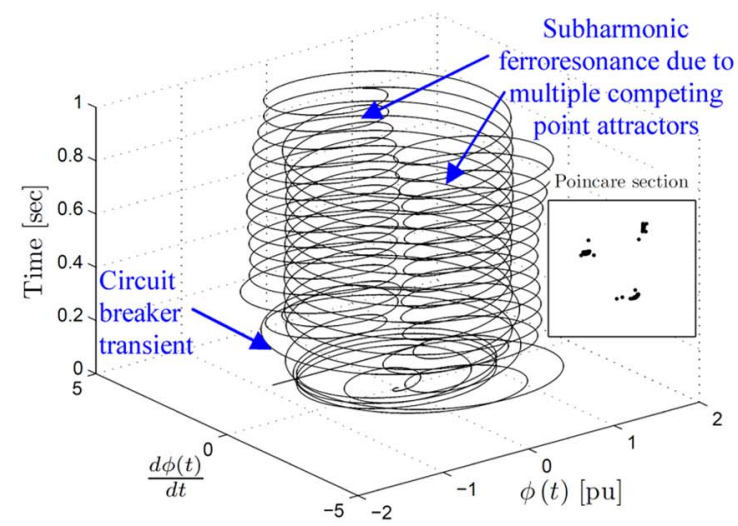

(b)

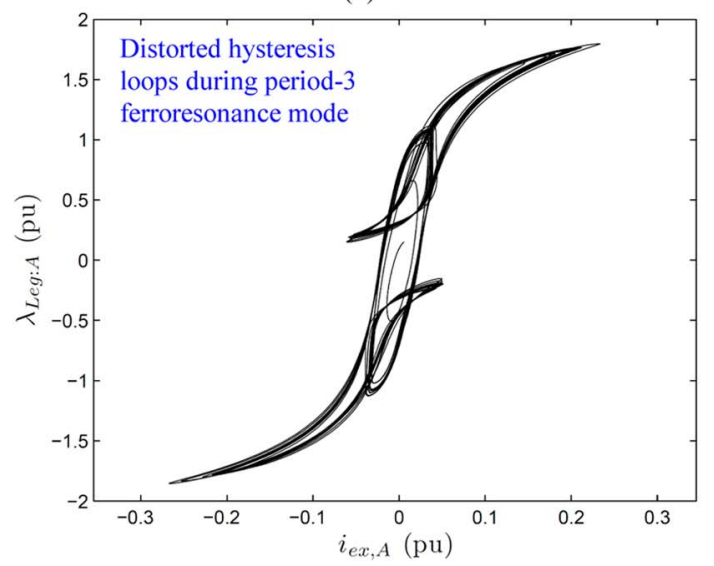

(c)

Fig. 7 (a) Time-domain waveforms of transformer primary voltages, winding currents and core fluxes for period-3 subharmonic ferroresonance mode $\left(C_{\text {shunt }}=4 \mu \mathrm{F}\right)$. (b) Phase-plane trajectory and (c) hysteresis loop (phase $a$ ) clearly indicates the transient caused by circuit breaker operation and the existence of competing attractors in the system orbits. The circuit breaker is opened at an initial phase angle of $\psi=90^{\circ}$ w.r.t. peak phase $a$ voltage.

inating magnetic leg couplings. The corresponding bifurcation diagram is very similar to Fig. 5 (not shown) and similar ferroresonance modes are predicted. However, on closer examination of time-domain waveforms (e.g., $C_{\text {shunt }}=5 \mu \mathrm{F}$ ), ignoring inter-phase magnetic couplings results in significant differences 

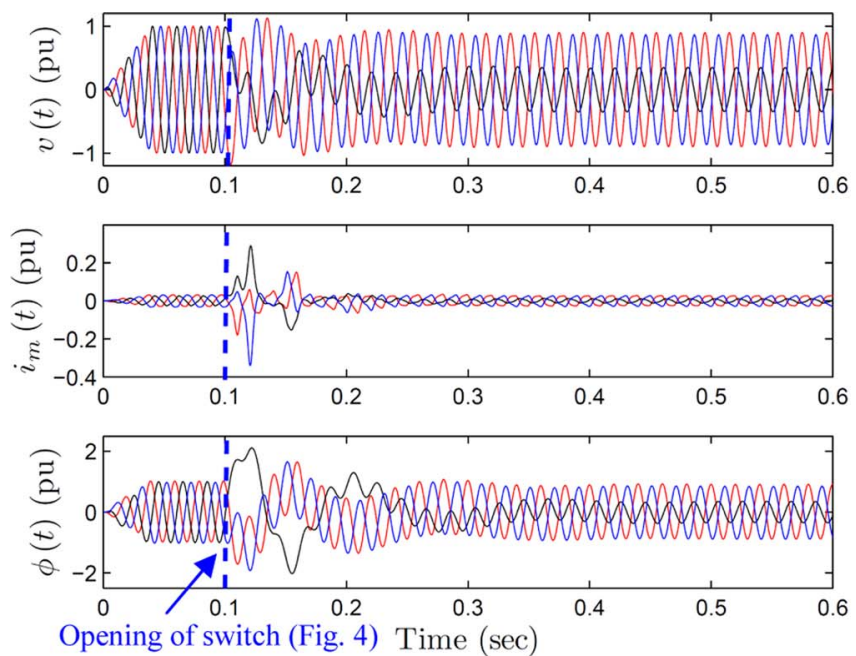

(a)

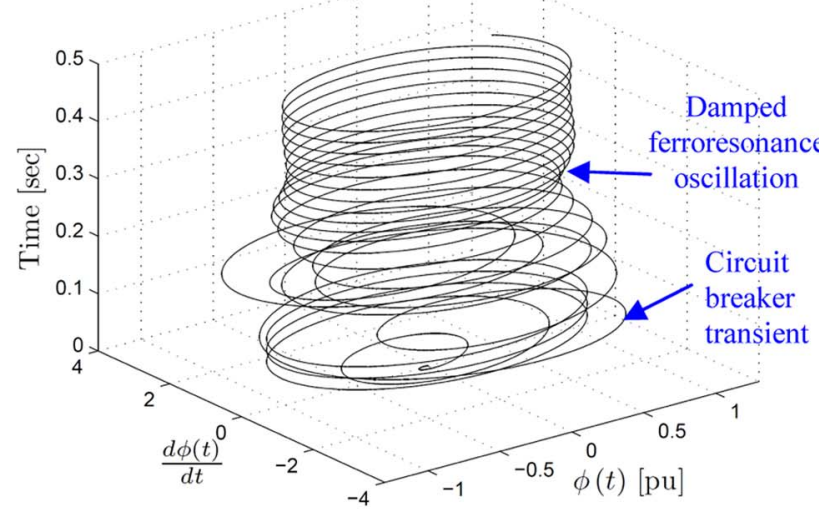

(b)

Fig. 8. (a) Time-domain waveforms of transformer voltages, winding currents, and fluxes for unstable ferroresonance. (b) Phase-plane trajectory indicates the transitory period and damping of ferroresonance in the steady state. The circuit breaker is opened at an initial phase angle of $\psi=60^{\circ}$ w.r.t. peak phase $a$ voltage.

in the predicted waveforms when compared to the proposed model (see Fig. 9). Furthermore, the computed phase-plane trajectories (drawn in 2 dimensions to highlight incongruities) assume different orbit trajectories.

\section{EXPERIMENTAL RESULTS AND MODEL VALIDATION}

Further investigations of three-phase transformer ferroresonance were carried out through experimental tests for the system of Fig. 4. The validity of the developed transformer model under ferroresonance conditions is examined by comparing simulations of ferroresonance with real measurements obtained from a dry-type three-leg 440/55-V laboratory test transformer (see Fig. 15 in the Appendix). The transformer electrical parameters have been determined from three-phase open and short circuit tests. The nonlinear asymmetric core magnetizing characteristics were measured by single-phase sinusoidal excitations of each leg. Based on the parameters given in the Appendix, the core models for each magnetic leg closely agree with mea-
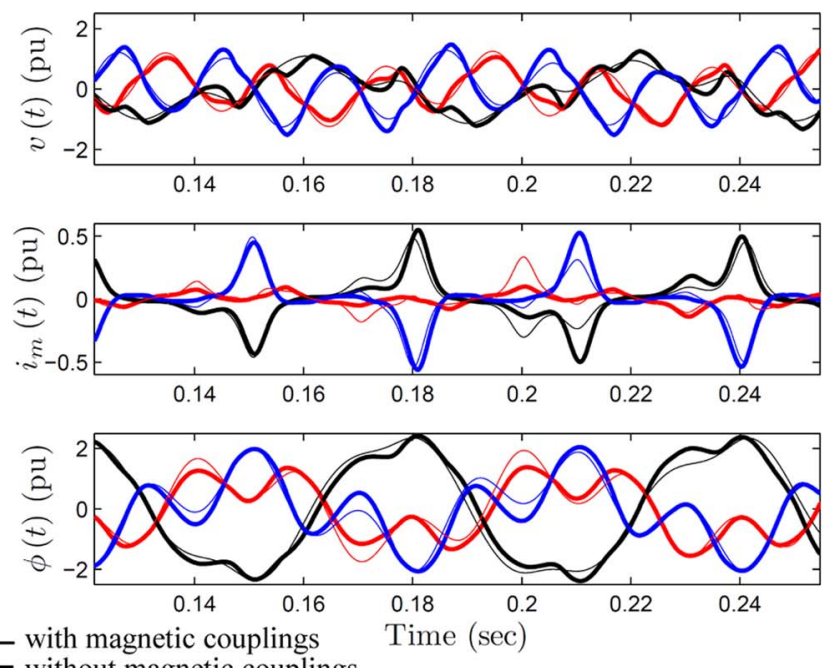

— with magnetic couplings

(a)

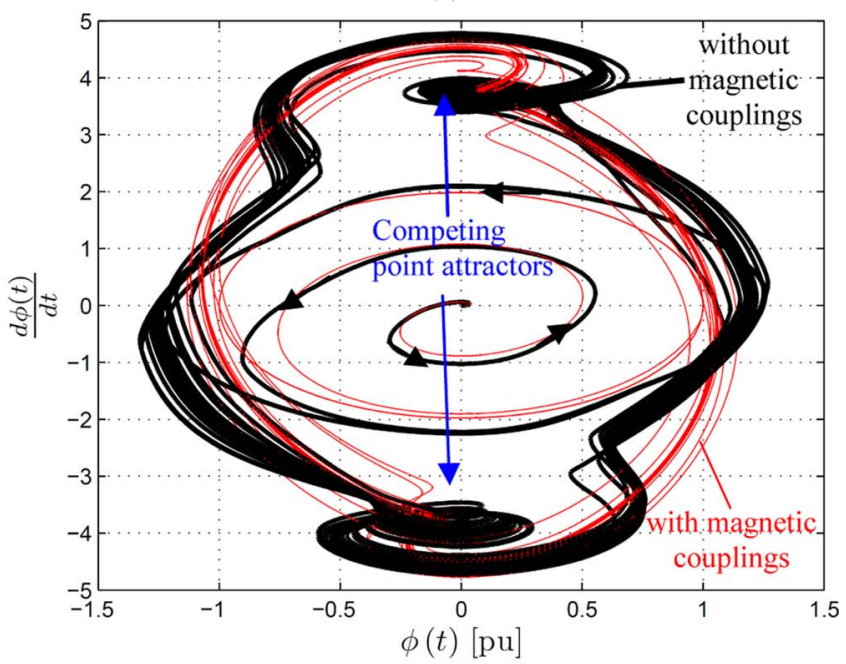

(b)

Fig. 9. Comparisons of simulated ferroresonance oscillations for $C_{\mathrm{shunt}}=$ $5 \mu \mathrm{F}$ considering core leg magnetic couplings (thin lines) versus three singlephase transformer bank with no magnetic couplings (thick lines). Time-domain waveforms (a) for voltages, currents, and fluxes are shown (see Fig. 11 for model comparison to measurements) and (b) phase-plane trajectories (drawn in two dimensions to highlight differences). Both models indicate period-3 subharmonic modes, however, wave shapes are incongruent and phase-plane trajectories diverge to different orbits.

sured hysteresis loops for a wide range of excitations (see Fig. 10).

\section{A. Measurement Apparatus and Method}

The three-phase experimental setup (see the Appendix) consists of an autotransformer source, isolation transformers, three single-phase circuit breakers for imposing unbalanced switching transients, capacitor banks, and a 440/55-V three-phase threeleg transformer. Each circuit breaker has a fixed measured opencircuit capacitance of approximately $30 \mathrm{pF}$. The switchable capacitor banks operate in the microfarad range and are connected in shunt arrangement, as shown in Fig. 4. Two synchronized oscilloscopes were setup to capture the transformer primary phase-to-neutral voltages and phase winding currents. 


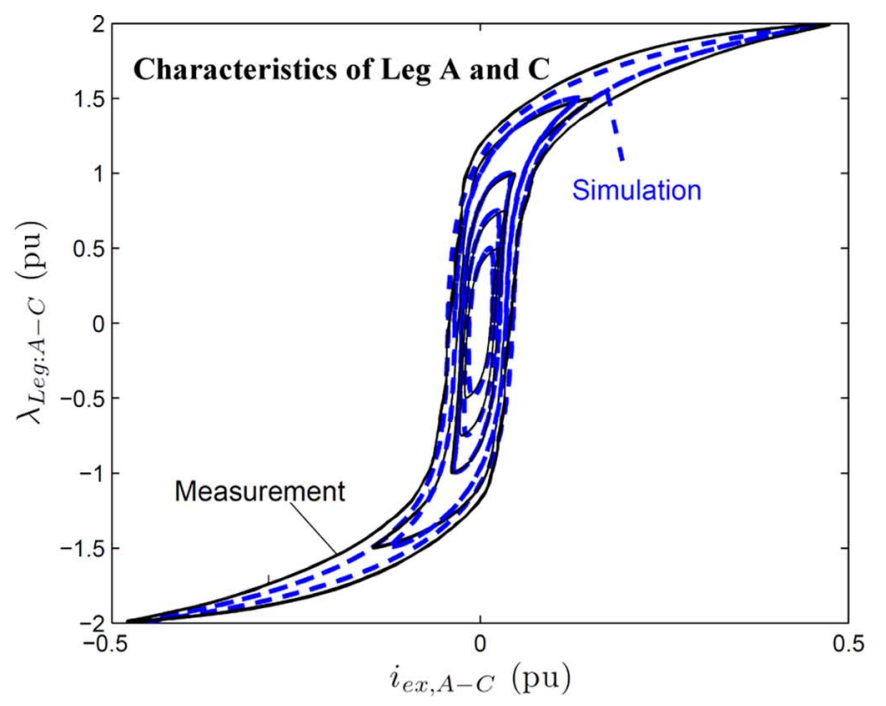

(a)

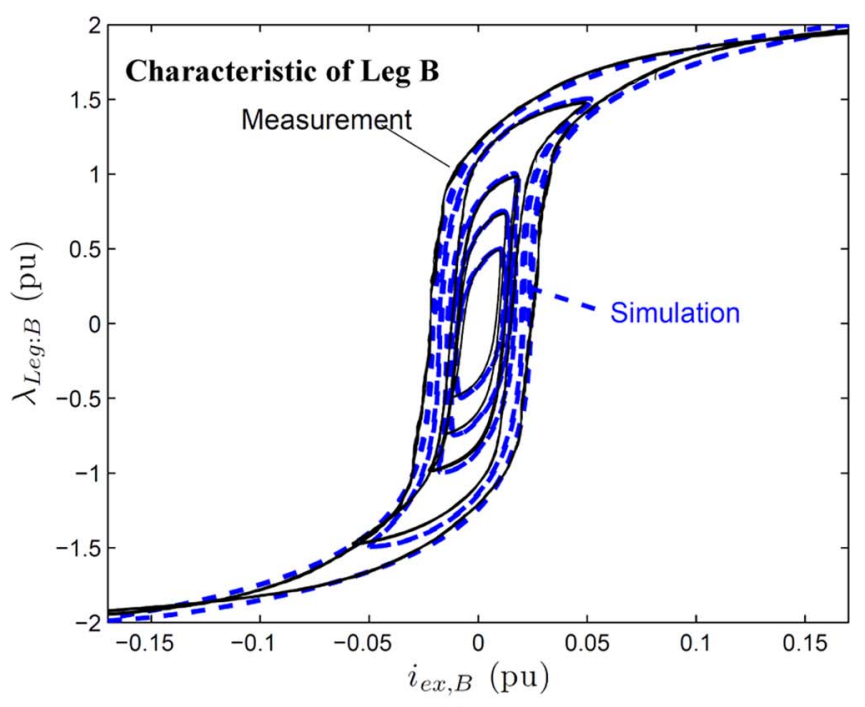

(b)

Fig. 10. Measured (solid line) versus modeled (dashed line) core magnetization hysteresis loops (major and minor) for (a) outer and (b) center legs at different excitation levels.

In order to obtain good comparisons of measured and simulated waveforms, the initial conditions (i.e., the instant of voltage phase angle the circuit breaker operates) at the onset of ferroresonance must be determined precisely. The slightest phase mismatch can lead to large discrepancies. The two oscilloscopes were triggered simultaneously at the moment of circuit breaker operation to capture initial conditions and subsequent ferroresonance oscillations. Measurements of ferroresonance were obtained with different shunt capacitance values and switching of phase $b$ circuit breaker.

\section{B. Experimental Results}

Fig. 11 displays the measured and model computed period-3 subharmonic ferroresonance modes in the primary voltage waveforms $\left(C_{\text {shunt }}=5 \mu \mathrm{F}\right)$. Close agreement is shown for the transition period (bifurcation) from normal conditions to fer-
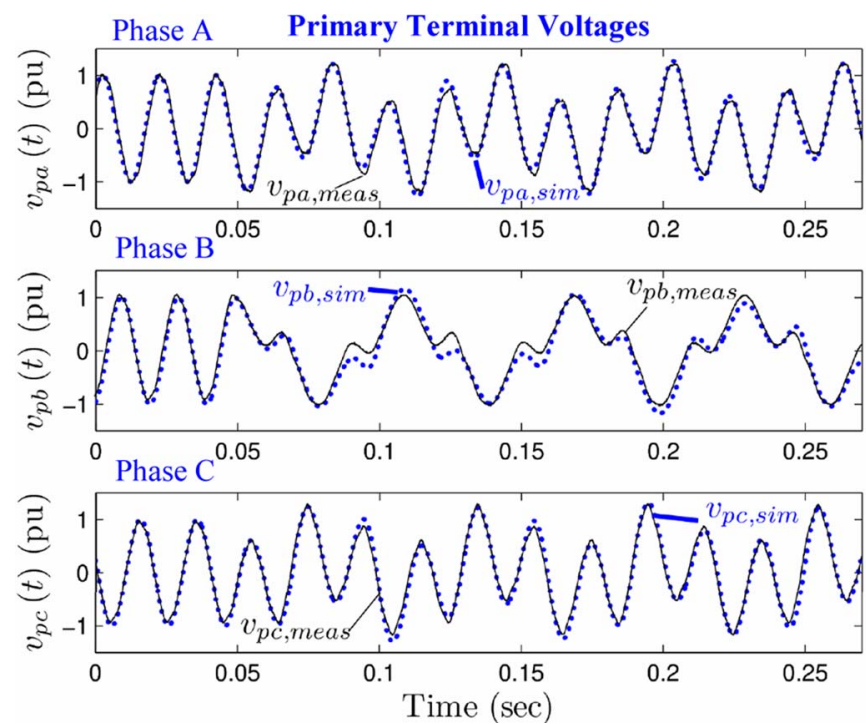

Fig. 11. Comparison of measured (solid line) versus simulated (dashed line) ferroresonance oscillations in transformer primary voltage $\left(v_{p}\right)$ waveforms $\left(C_{\text {shunt }}=5 \mu \mathrm{F}\right)$. Transition (bifurcation) from normal operation to stable period-3 subharmonic ferroresonance (bifurcation) is shown. The circuit breaker is opened at an initial phase angle of $\psi=-137^{\circ}$ w.r.t. peak phase $a$ voltage.

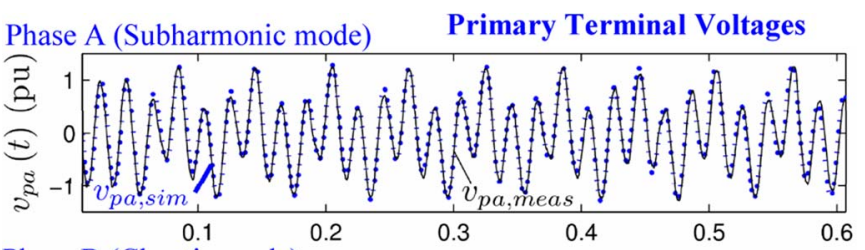

Phase B (Chaotic mode)
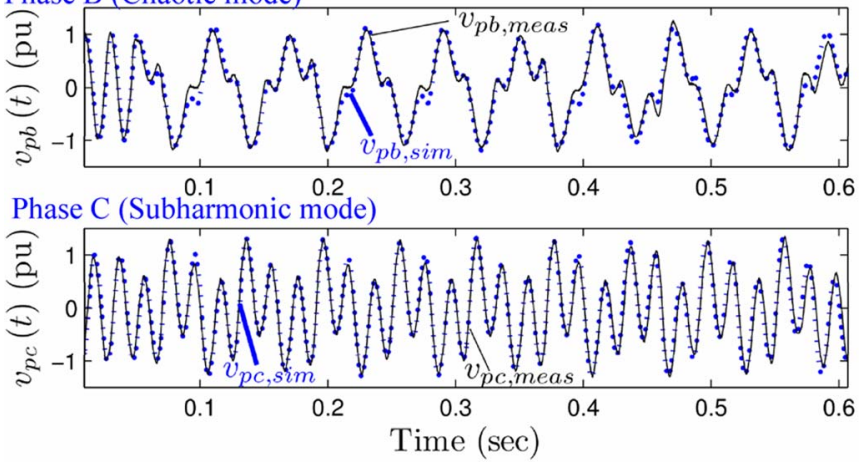

Fig. 12. Comparison of measured (solid line) versus simulated (dashed line) chaotic ferroresonance oscillations in transformer primary voltage $\left(v_{p}\right)$ waveforms $\left(C_{\text {shunt }}=7 \mu \mathrm{F}\right)$. Stable subharmonic period-3 (phases $a$ and $c$ ) and chaotic ferroresonance oscillations (phase $b$ ) are shown. The circuit breaker is opened at an initial phase angle of $\psi=-74^{\circ}$ w.r.t. peak phase $a$ voltage.

roresonance as well as the subsequent steady-state oscillations in all three phases. Note that the wave shapes are not identical amongst all phases. The wave shape of the faulted phase $b$ is markedly different to phases $a$ and $c$, but is still period-3 mode. This is because the ferroresonance path of the center leg is not identical to the other legs due to the open phase $b$ condition. The center phase magnetizing inductance has a direct path to the shunt capacitance compared to the other two phases (see Fig. 4). Furthermore, the core is asymmetric because the center leg (phase $b$ ) has a shorter flux path length. Since phase $b$ is open, the voltage is not being supplied by the source, but 


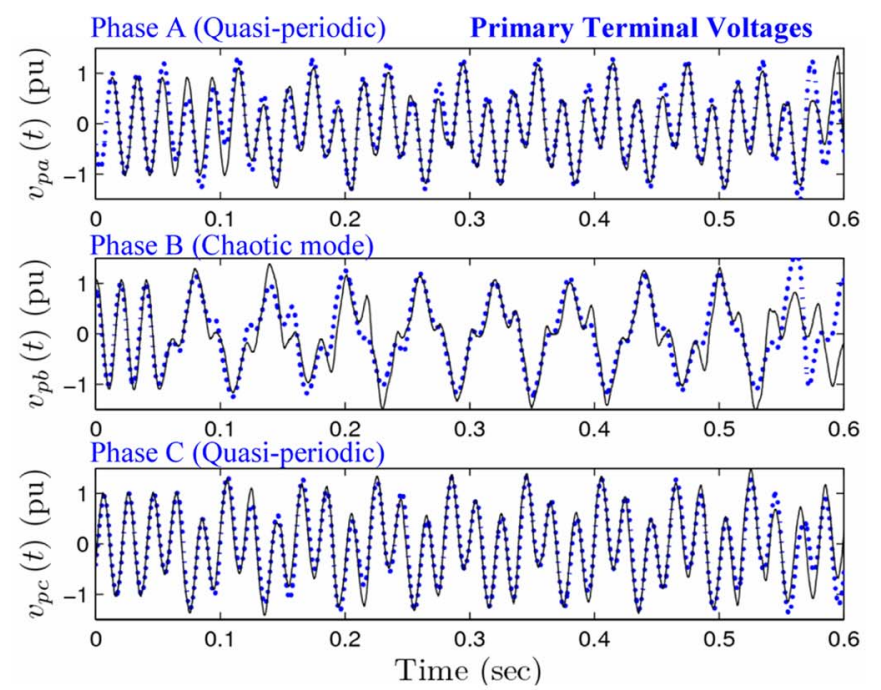

(a)
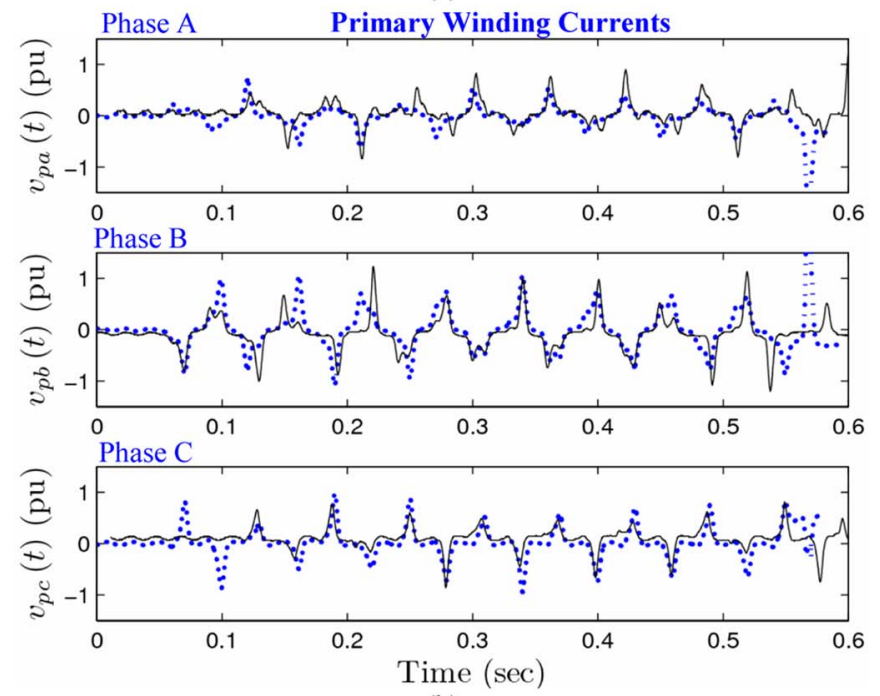

(b)

Fig. 13. Comparison of measured (solid line) versus simulated (dashed line) chaotic ferroresonance oscillations for primary (a) terminal voltages $\left(v_{p}\right)$ and (b) winding currents $\left(i_{p}\right)$ for $C_{\text {shunt }}=9 \mu \mathrm{F}$. The waveforms exhibit chaotic behavior in phase $b$ and quasi-periodic mode resembling subharmonic (period-3) ferroresonance in phases $a$ and $c$. The circuit breaker is opened at an initial phase angle of $\psi=60^{\circ}$ w.r.t. peak phase $a$ voltage. The errors are due to the sensitivity of chaos to mismatching of initial conditions.

is developed by induction from magnetic flux couplings of the core.

Comparisons of measured and simulated waveforms for $C_{\text {shunt }}=7 \mu \mathrm{F}$ are shown in Fig. 12. It is interesting to note that phases $a$ and $c$ exhibit subharmonic ferroresonance oscillations while phase $b$ waveforms are chaotic. This has not been documented in prior studies. This behavior is due to the complicated electromagnetic phase couplings in the core. The developed transformer model accurately duplicates this behavior under the same conditions. Further experimental tests determined more chaotic modes for larger $C_{\text {shunt }}$ values.

Fig. 13 depicts the measured and simulated phase voltages and winding currents for $C_{\text {shunt }}=9 \mu \mathrm{F}$. Chaotic ferroresonance modes are observed in phase $b$; however, phases $a$ and $c$ waveforms resemble period- 3 mode but are strictly
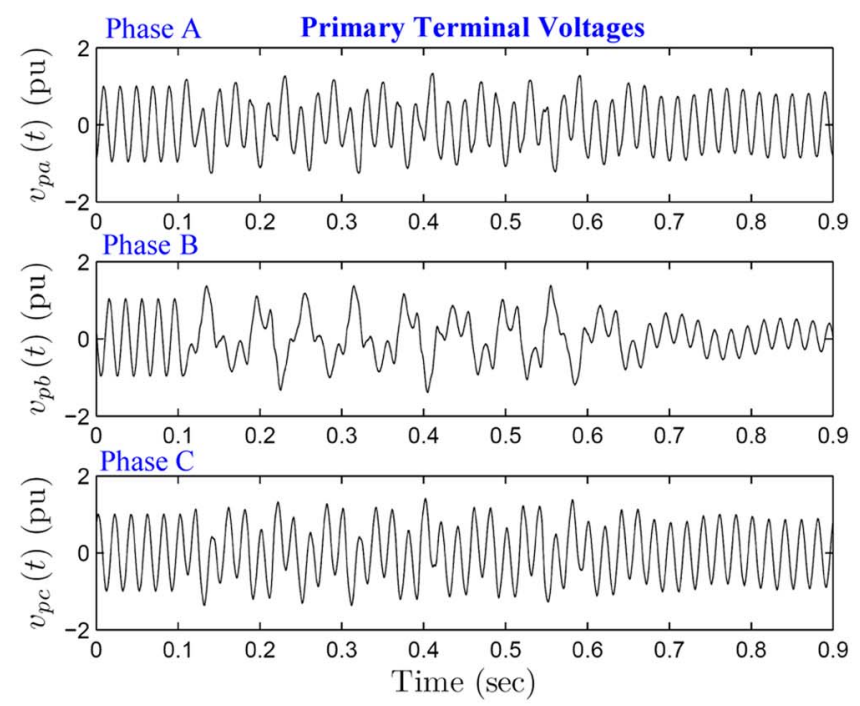

(a)
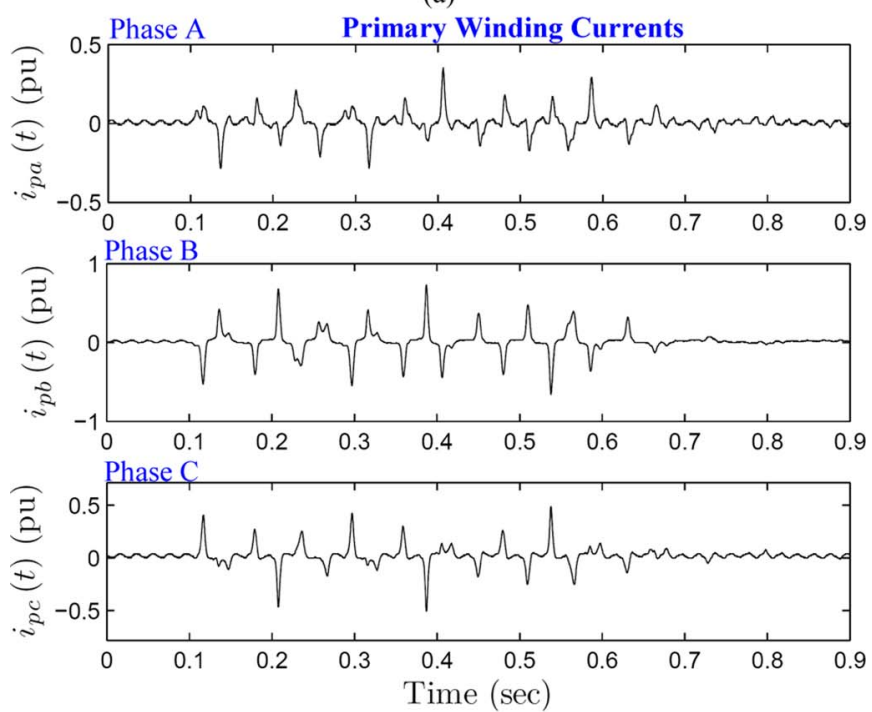

(b)

Fig. 14. Measured transformer waveforms for primary (a) terminal voltages $\left(v_{p}\right)$ and (b) winding currents $\left(i_{p}\right)$. The previous case (see Fig. 13) is repeated for the same $C_{\text {shunt }}(9 \mu \mathrm{F})$ but at a different circuit breaker opening time which results in momentary unstable chaotic ferroresonance. The circuit breaker is opened at an initial phase angle of $\psi=66^{\circ}$ w.r.t. peak phase $a$ voltage.

nonrepeating (quasi-periodic). The maximum peak voltage is approximately $1.5 \mathrm{pu}$ for $C_{\text {shunt }}$ between 8.0 and $9.5 \mu \mathrm{F}$. These high overvoltages can degrade winding insulation and cause excessive losses.

Note that after a few cycles, the simulated chaotic waveforms tend to diverge away from measurements due to sensitivity to small unaccounted for perturbations (e.g., supply harmonics) and differences in initial conditions (e.g., precise switching instant). A summary of the measured ferroresonance modes and peak phase voltages is shown in Table I.

Lastly, the influence of circuit breaker switching time on resulting ferroresonance behavior was examined experimentally (see Fig. 14). The case for $C_{\text {shunt }}=9 \mu \mathrm{F}$ (see Fig. 13) was repeated for a different switching angle with respect to phase $a$ voltage peak. By changing the circuit breaker opening instant 
TABLE I

Observed Ferroresonance Modes and Peak Phase Voltage VALUES: $C_{\text {series }}=30 \mathrm{pF}$ AND $C_{\text {shunt }}=2$ TO $10 \mu \mathrm{F}$

\begin{tabular}{lcccccc}
\hline \hline $\begin{array}{c}\mathrm{C}_{\text {shunt }} \\
(\mu \mathrm{F})\end{array}$ & \multicolumn{2}{c}{ Ferroresonance Modes* } & $\begin{array}{c}V_{a}^{\text {peak }} \\
\text { Phase } a\end{array}$ & $\begin{array}{c}\text { Phase } b \\
\text { Phase } c\end{array}$ & $\begin{array}{c}V_{b}^{\text {peak }} \\
(\mathrm{pu})\end{array}$ & $\begin{array}{c}V_{c}^{\text {peak }} \\
(\mathrm{pu})\end{array}$ \\
\hline 2.0 & I & I & I & 1.16 & 0.82 & 1.16 \\
3.0 & I & I & I & 1.17 & 1.04 & 1.29 \\
4.0 & III & III & III & 1.25 & 1.17 & 1.40 \\
5.0 & III & III & III & 1.20 & 1.05 & 1.37 \\
5.5 & III & III & III & 1.21 & 1.09 & 1.37 \\
6.0 & III & C & III & 1.29 & 1.30 & 1.43 \\
6.5 & III & C & III & 1.35 & 1.25 & 1.45 \\
7.0 & III & C & III & 1.27 & 1.27 & 1.40 \\
7.5 & III & C & III & 1.38 & 1.35 & 1.46 \\
8.0 & III & C & III & 1.45 & 1.45 & 1.49 \\
8.5 & III & C & III & 1.43 & 1.37 & 1.49 \\
9.0 & Q-III & C & Q-III & 1.45 & 1.43 & 1.48 \\
9.5 & Q-III & C & Q-III & 1.40 & 1.49 & 1.45 \\
10.0 & Q-III & C & Q-III & 1.43 & 1.45 & 1.45 \\
\hline \hline
\end{tabular}

*) Ferroresonance modes- I: period-1 fundamental, III: period-3 subharmonic, Q: quasi-periodic, C: chaotic, Q-III: quasi-periodic modes resembling period-3 type ferroresonance.

from $\psi=60^{\circ}$ to $\psi=66^{\circ}$, the previously stable chaotic modes became unstable and dampened out.

\section{CONCLUSION}

A new analysis of the stability domain of three-phase transformer ferroresonance in multi-leg core structures is presented in this paper. For the first time, a newly developed threeleg core model considering dynamic leg hysteresis nonlinearities, core topology and asymmetric leg couplings is applied to ferroresonance. Extensive experimental tests of transformer ferroresonance have been performed to confirm the accuracy of model predictions. The main conclusions are as follows.

1) The study results indicates that the stability domain of ferroresonance modes and amplitude oscillations (e.g., voltage, current and fluxes) are highly dependent on the correct modeling of magnetic couplings and nonlinearities of the iron-core structure.

2) Predicted ferroresonance modes (e.g., subharmonic and chaotic modes) are shown to be misrepresented in threephase transformer models that neglect hysteresis nonlinearities (e.g., using single-value functions). False ferroresonance modes and the omission of more severe chaotic modes are shown for nonhysteretic core models.

3) Likewise, the magnetic couplings in the legs are shown to significantly impact the accuracy of predicted wave shapes and trajectories of ferroresonance oscillations.

4) For the first time, measured and modeled ferroresonance oscillations are shown to exhibit different behavior in each phase. For particular conditions, chaotic voltage waveforms were observed in the open circuited phase and subharmonic or quasi-periodic modes in the other phases. Therefore, extending per-phase modeling approaches and analysis techniques to the study of three-phase transformer ferroresonance behavior can lead to incorrect results.

5) The developed three-leg core model correctly predicted ferroresonance modes observed in laboratory tests. Results show that the model duplicates the three-phase terminal voltage waveforms with high accuracy under transient and steady-state ferroresonance conditions.

6) Moreover, for stable periodic modes (e.g., subharmonics), the model accurately predicted their existence for the range of capacitance values observed experimentally. For nonperiodic modes (e.g., quasi-periodic and chaotic), the model can reproduce waveforms accurately for a few cycles after the bifurcation point if initial conditions are known precisely. However, due sensitivity of chaos to minute differences in initial conditions (e.g., switching transient) and small unaccountable perturbations (e.g., supply harmonics), incorrect predictions occur for long term behavior under chaotic conditions.

7) The proposed three-leg transformer modeling approach is general and expandable to the study of ferroresonance and other transient disturbances in different electrical machines, transformer types and configurations (e.g., fiveleg transformers, induction machines, etc.). The modeling approach is useful for evaluating transformer stresses (e.g., insulation) and testing dynamics of three-leg core designs.

\section{APPENDIX}

\section{Model PARAMETERS AND EXPERIMENTAL SETUP}

TABLE II

Hysteresis AND MAGNETIC CORE MODEl DATA

\begin{tabular}{ll}
\hline \hline Ascending function $\phi_{x}^{+}\left(f_{x}\right)$ & Descending function $\phi_{x}^{-}\left(f_{x}\right)$ \\
\hline$\alpha_{a}=0.73, \beta_{a}=300, \sigma_{a}=0.2$ & $\alpha_{a}=0.73, \beta_{a}=300, \sigma_{a}=0.1$ \\
$\alpha_{b}=0.65, \beta_{b}=1400, \sigma_{b}=0.1$ & $\alpha_{b}=0.68, \beta_{b}=1400, \sigma_{b}=0.01$ \\
$\alpha_{c}=0.73, \beta_{c}=300, \sigma_{c}=0.2$ & $\alpha_{c}=0.73, \beta_{c}=300, \sigma_{c}=0.1$ \\
\hline Magnetic parameters: $\Re_{0}=1500 \mathrm{A.t} / \mathrm{Wb}, \rho_{a}=0.1, \rho_{b}=1.0, \rho_{c}=0.1$ \\
\hline
\end{tabular}

TABLE III

TRANSFORMER DATA AND ELECTRIC EQUIVALENT CiRCUIT PARAMETERS

\begin{tabular}{ll}
\hline \hline \multicolumn{1}{c}{ Description } & \multicolumn{1}{c}{ Data } \\
\hline Rated primary winding voltage $\left(v_{p}\right)$ & $440 \mathrm{~V}$ \\
Rated secondary winding voltage $\left(v_{s}\right)$ & $55 \mathrm{~V}$ \\
Turns ratio $(N)$ & 8 \\
Operating frequency $(f)$ & $50 \mathrm{~Hz}$ \\
Rated secondary current $\left(i_{s}\right)$ & $10 \mathrm{~A}$ \\
Primary winding resistance $\left(R_{p a,}, R_{p b}, R_{p c}\right)$ & $9.423 \Omega(0.0268 \mathrm{pu})$ \\
Secondary winding resistance $\left(R_{s a}, R_{s b}, R_{s c}\right)$ & $0.147 \Omega(0.0268 \mathrm{pu})$ \\
Primary leakage inductance $\left(L_{p a}, L_{p b}, L_{p c}\right)$ & $6.341 \mathrm{mH}(0.00566 \mathrm{pu})$ \\
Secondary leakage inductance $\left(L_{s a}, L_{s b}, L_{s c}\right)$ & $0.0991 \mathrm{mH}(0.00566 \mathrm{pu})$ \\
\hline \hline
\end{tabular}

$Z_{\mathrm{p}, \text { base }}=N^{2} \cdot Z_{\mathrm{s}, \text { base }}=N^{2} \cdot v_{\mathrm{s}, \text { (rated) }} i_{\mathrm{s}, \text { (rated) }}($ primary referred). 


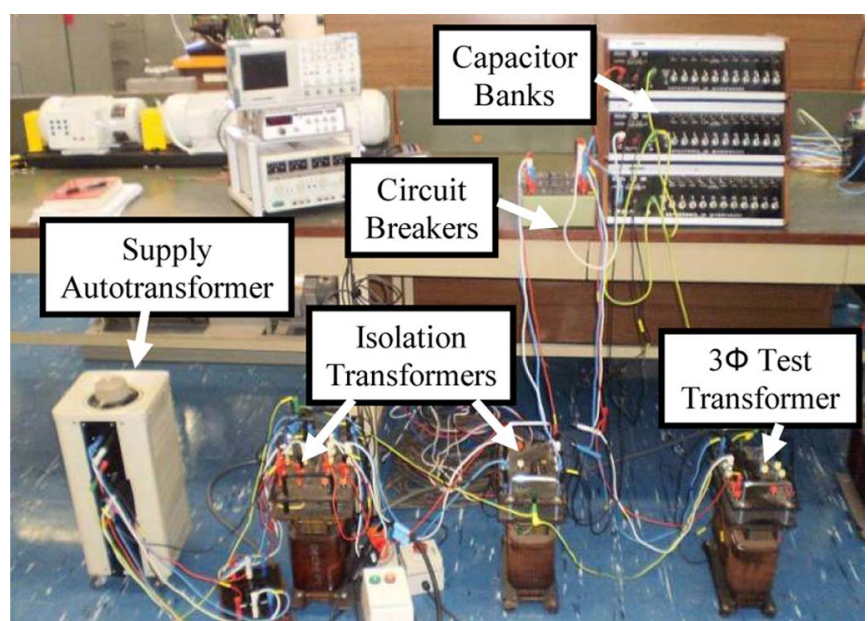

Fig. 15. Experimental setup for three-phase transformer ferroresonance tests.

\section{REFERENCES}

[1] E. F. Fuchs and M. A. S. Masoum, Power Quality in Power Systems and Electrical Machines. Amsterdam, CA, The Netherlands: Elsevier, 2008.

[2] M. R. Iravani, A. K. S. Chaudhary, W. J. Giesbrecht, I. E. Hassan, A. J. F. Keri, K. C. Lee, J. A. Martinez, A. S. Morched, B. A. Mork, M. Parniani, A. Sharshar, D. Shirmohammadi, R. A. Walling, and D. A. Woodford, "Modeling and analysis guidelines for slow transients-Part III. The study of ferroresonance," IEEE Trans. Power Deliv., vol. 15, no. 1, pp. 255-265, Jan. 2000

[3] P. Ferracci, "Ferroresonance," Group Schneider: Cahier No. 190, pp. 128, Mar. 1998.

[4] R. C. Dugan, "Examples of ferroresonance in distribution," in Proc. IEEE Power Eng. Soc. General Meet., 2003, vol. 2, pp. 1213-1215.

[5] D. A. N. Jacobson, "Examples of ferroresonance in a high voltage power system," in Proc. IEEE Power Eng. Soc. General Meet., 2003, vol. 2, pp. 1206-1212.

[6] K. Laohacharoensombat, K. Tuitemwong, S. Jaruwattanadilok, C. Wattanasakpubal, and K. Kleebmek, "Case study of ferroresonance in $33 \mathrm{kV}$ distribution network of PEA Thailand," in Proc. IEEE Reg. 10 Conf. (TENCON)., 2004, vol. C, pp. 417-420.

[7] P. E. Sutherland and R. Manning, "Ferroresonance in a 13.8 kV Distribution Line," in Proc. 41st IAS Annu. Meet. IEEE Ind. Appl. Conf., 2006, pp. 2238-2241.

[8] T. Tsao and C. Ning, "Analysis of ferroresonant overvoltages at maanshan nuclear power station in taiwan," IEEE Trans. Power Deliv., vol. 21, no. 2, pp. 1006-1012, Apr. 2006.

[9] V. Simha and W. Lee, "The jump phenomena," IEEE Industry Appl. Mag., vol. 14 , no. 5, pp. 53-59, Oct. 2008.

[10] A. Clerici and C. H. Didriksen, "Dynamic overvoltages and ferroresonance found in switching surge studies for Iran $400 \mathrm{kV}$ system," IEEE Trans. Power Apparatus Syst., vol. PAS-91, no. 1, pp. 195-203, Jan. 1972.

[11] D. R. Crane and G. W. Walsh, "Large mill power outages caused by potential transformer ferroresonance," IEEE Trans. Ind. Appl., vol. 24, no. 4, pp. 635-640, Jul./Aug. 1988

[12] J. Bethenod, "Sur le transformateur et résonance," L'Eclairae Electrique, pp. 289-296, 1907.

[13] P. Boucherot, "Existence de deux régimes en ferro-résonance," R. G. E., pp. 289-296, 1920.

[14] J. W. Butler and C. Concordia, "Analysis of series capacitor application problems," AIEE Trans, vol. 56, pp. 975-988, 1937.

[15] R. H. Hopkinson, "Ferroresonance during single-phase switching of 3phase distribution transformer banks," IEEE Trans. Power Apparatus Syst., vol. 84, no. 4, pp. 289-293, Apr. 1965.

[16] L. B. Crann and R. B. Flickinger, "Overvoltages on 14.4/24.9-kV rural distribution systems," AIEE Trans. Power Apparatus Syst., vol. 73, pp. 1208-1212, 1954.

[17] G. G. Auer and A. J. Schultz, "An analysis of 14.4/24.9-kV grounded-wye distribution system overvoltages," AIEE Trans. Power Apparatus Syst., vol. 73, pp. 1027-1032, 1954.
[18] C. Hayashi, Nonlinear Oscillations in Physical Systems. New York, NY: McGraw-Hill, 1964.

[19] R. Rudenberg, Transient Performance of Electric Power Systems. New York, NY: McGraw-Hill, 1950.

[20] C. Kieny, "Application of the bifurcation theory in studying and understanding the global behavior of a ferroresonant electric power circuit," IEEE Trans. Power Deliv., vol. 6, no. 2, pp. 866-872, Apr. 1991.

[21] B. A. Mork and D. L. Stuehm, "Application of nonlinear dynamics and chaos to ferroresonance in distribution systems," IEEE Trans. Power Deliv., vol. 9, no. 2, pp. 1009-1017, Apr. 1994.

[22] J. M. T. Thompson and H. B. Stewart, Nonlinear Dynamics and Chaos : Geometrical Methods for Engineers and Scientists. West Sussex, U.K. Wiley, 1986.

[23] F. Wornle, D. K. Harrison, and Z. Chengke, "Analysis of a ferroresonan circuit using bifurcation theory and continuation techniques," IEEE Trans. Power Deliv., vol. 20, no. 1, pp. 191-196, Jan. 2005.

[24] A. Ben-Tal, D. Shein, and S. Zissu, "Studying ferroersonance in actual power systems by bifurcation diagram," Electric Power Syst. Res., vol. 49 , pp. 175-183, 1999.

[25] G. Mokryani, M. R. Haghifam, H. Latafat, P. Aliparast, and A. Abdollahy, "Analysis of ferroresonance in a $20 \mathrm{kV}$ distribution network," in Proc. 2nd Int. Conf. Power Electron. Intell. Transportation Syst. (PEITS), 2009, pp. 31-35.

[26] B. Tanggawelu, R. N. Mukerjee, and A. E. Ariffin, "Ferroresonance studies in Malaysian utility's distribution network," in Proc. IEEE Power Eng. Soc. Gen. Meet., 2003, vol. 2, pp. 1216-1219.

[27] G. Mokryani and M. R. Haghifam, "Application of wavelet transform and MLP neural network for ferroresonance identification," Proc. IEEE Power Energy Soc. Gen. Meet., pp. 1-6, 2008.

[28] B. Zhang and T. Lu, "On the use of wavelet decomposition for ferroresonance detection in power system," in Proc. Asia-Pacific Power Energy Eng. Conf. (APPEEC), 2009, pp. 1-4.

[29] W. Piasecki, M. Florkowski, M. Fulczyk, P. Mahonen, and W. Nowak, "Mitigating ferroresonance in voltage transformers in ungrounded MV networks," IEEE Trans. Power Deliv., vol. 22, no. 4, pp. 2362-2369, Oct. 2007.

[30] P. Picher, L. Bolduc, B. Girard, and V. N. Nguyen, "Mitigation of ferroresonance induced by single-phase opening of a three-phase transformer feeder," in Proc. Can. Conf. Electr. Comput. Eng. (CCECE), 2006, pp. $482-485$.

[31] M. Sanaye-Pasand, A. Rezaei-Zare, H. Mohseni, S. Farhangi, and R. Iravani, "Comparison of performance of various ferroresonance suppressing methods in inductive and capacitive voltage transformers," presented at the IEEE Power India Conf., New Delhi, India, 2006.

[32] Y. Yu and H. Zhou, "Study on simulation of ferroresonance elimination in $10 \mathrm{kV}$ power system," in Proc. IEEE/PES Transm. Distrib. Conf. Exhibition: Asia Pac., 2005, pp. 1-7.

[33] L. Yunge, S. Wei, Q. Rui, and Y. Jilin, "A systematical method for suppressing ferroresonance at neutral-grounded substations," IEEE Trans. Power Deliv., vol. 18, no. 3, pp. 1009-1014, Jul. 2003.

[34] P. G. Khorasani and A. Deihimi, "A new modeling of Matlab transformer for accurate simulation of ferroresonance," in Proc. Int. Conf. Power Eng., Energy Electr. Drives (POWERENG), 2009, pp. 529-534.

[35] P. S. Moses and M. A. S. Masoum, "Modeling ferroresonance in asymmetric three-phase power transformers," in Proc. Australas. Univ. Power Eng. Conf. (AUPEC), 2009, pp. 1-6.

[36] A. V. Makarov and V. G. Komin, "The research of ferroresonant phenomena in electric circuits under open-phase operating conditions," in Proc. IEEE Russia Power Tech., 2005, pp. $1-7$.

[37] B. C. Lesieutre, J. A. Mohamed, and A. M. Stankovic, "Analysis of ferroresonance in three-phase transformers," in Proc. Int. Conf. Power Syst. Tech. (PowerCon)., 2000, vol. 2, pp. 1013-1018.

[38] A. Tokic, V. Madzarevic, and I. Uglesic, "Numerical calculations of threephase transformer transients," IEEE Trans. Power Deliv., vol. 20, no. 4, pp. 2493-2500, 2005.

[39] K. Okumura, "Nonlinear oscillation of three-phase circuit," Electr. eng. Jpn, vol. 96, pp. 106-112, 1976.

[40] H. Lamba, M. Grinfeld, S. McKee, and R. Simpson, "Subharmonic ferroresonance in an LCR circuit with hysteresis," IEEE Trans. Magnetics, vol. 33, no. 4, pp. 2495-2500, Jul. 1997. 
[41] A. Rezaei-Zare, R. Iravani, and M. Sanaye-Pasand, "Impacts of transformer core hysteresis formation on stability domain of ferroresonance modes," IEEE Trans. Power Deliv., vol. 24, no. 1, pp. 177-186, Jan. 2009.

[42] S. K. Chakravarthy and C. V. Nayar, "Parallel (quasi-periodic) ferroresonant oscillations in electrical power systems," IEEE Trans. Circuits Systems I: Fundam. Theory Appl., vol. 42, no. 9, pp. 530-534, Sep. 1995.

43] D. A. N. Jacobson, P. W. Lehn, and R. W. Menzies, "Stability domain calculations of period-1 ferroresonance in a nonlinear resonant circuit," IEEE Trans. Power Deliv., vol. 17, no. 3, pp. 865-871, Jul. 2002.

[44] L. Yunge, S. Wei, and L. Furong, "Novel analytical solution to fundamental ferroresonance-part I: Power frequency excitation characteristic," IEEE Trans. Power Syst., vol. 21, no. 2, pp. 788-793, Apr. 2006.

[45] L. Yunge, S. Wei, and L. Furong, "Novel analytical solution to fundamental ferroresonance-Part II: Criterion and elimination," IEEE Trans. Power Deliv., vol. 21, no. 2, pp. 794-800, Apr. 2006

[46] P. S. Moses, M. A. S. Masoum, and H. A. Toliyat, "Dynamic modeling of three-phase asymmetric power transformers with magnetic hysteresis: no-load and inrush conditions," IEEE Trans. Energy Convers., vol. 25 no. 4, pp. 1040-1047, Dec. 2010.

[47] E. C. Cherry, "The duality between interlinked electric and magnetic circuits and the formation of transformer equivalent circuits," Proc. Phys. Soc., vol. 62, pp. 101-111, 1949.

[48] M. A. S. Masoum and P. S. Moses, "Impact of balanced and unbalanced direct current bias on harmonic distortion generated by asymmetric threephase three-leg transformers," IET Electric Power Appl., vol. 4, pp. 507515,2010

[49] M. A. S. Masoum, P. S. Moses, and A. S. Masoum, "Derating of asymmetric three-phase transformers serving unbalanced nonlinear loads," IEEE Trans. Power Deliv., vol. 23, no. 4, pp. 2033-2041, Oct. 2008.

[50] J. Tellinen, "A simple scalar model for magnetic hysteresis," IEEE Trans. Magnetics, vol. 34, no. 4, pp. 2200-2206, Jul. 1998.

[51] E. F. Fuchs and Y. Yiming, "Measurement of $\lambda$-i characteristics of asymmetric three-phase transformers and their applications," IEEE Trans. Power Deliv., vol. 17, no. 4, pp. 983-990, Oct. 2002.

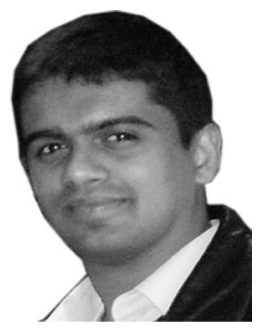

Paul S. Moses (S'09) received the B.Eng. (FirstClass Hons.) and B.Sc. degrees in electrical engineering and physics in 2006 from Curtin University, Perth, WA, Australia, where he is currently working toward the Ph.D. degree in electrical engineering.

Since 2007, he has also been a Research Scientist for the Defence Science and Technology Organization (DSTO), Department of Defence, HMAS Stirling, Australia, and is presently part of their Maritime Platforms Division, Propulsion and Energy Systems Group. His research interests include nonlinear electromagnetic phenomena, power quality, and protection.

Mr. Moses was the recipient of the W. J. Smith Memorial Prize for Best Electrical Engineering Honors Thesis and the Don Watts Prize from the Curtin Faculty of Engineering for the most innovative research project, and an Australian Postgraduate Award scholarship in 2009.

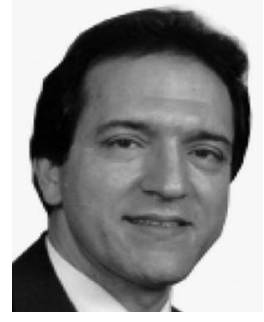

Mohammad A. S. Masoum (S'88-M'91-SM'05) received the B.S., M.S., and Ph.D. degrees in electrical and computer engineering in 1983, 1985, and 1991, respectively, from the University of Colorado, Boulder.

He is currently an Associate Professor and the discipline leader for electrical power engineering at the Electrical and Computer Engineering Department, Curtin University, Perth, Australia. His current research interests include optimization, power quality and stability of power systems/electric machines, and distributed generation. He is the coauthor of Power Quality in Power Systems and Electrical Machines (Elsevier, 2008).

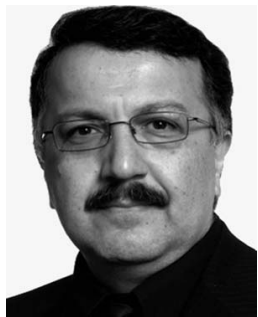

Hamid A. Toliyat (S'87-M'91-SM'96-F'08) received the B.S. degree from Sharif University of Technology, Tehran, Iran, in 1982, the M.S. degree from West Virginia University, Morgantown, in 1986, and the Ph.D. degree from the University of Wisconsin-Madison, Madison, in 1991, all in electrical engineering.

He is currently the Raytheon Endowed Professor at Texas A\&M University, College Station. He is the author of DSP-Based Electromechanical Motion Control (CRC, 2003) and the co-editor of Handbook of Electric Motors-2nd Edition (Marcel Dekker, 2004).

Mr. Toliyat has served as an Editor of the IEEE TRANSACTIONS ON ENERGY CONVERSION. He was the recipient of the following awards: the prestigious Cyrill Veinott Award in Electromechanical Energy Conversion from the IEEE Power Engineering Society in 2004; the Texas Engineering Experiment Station Fellow Award in 2004 and 2006; the Outstanding Professor Award in 2005, the Distinguished Teaching Award in 2003, the E.D. Brockett Professorship Award in 2002, the Eugene Webb Faculty Fellow Award in 2000, and the Texas A\&M Select Young Investigator Award in 1999, all from Texas A\&M University; the Space Act Award from the National Aeronautics and Space Administration in 1999; the Schlumberger Foundation Technical Award in 2001 and 2000; the Prize Paper Award from the IEEE Power Engineering Society in 1996 and 2006; and Third Prize Paper Award from the IEEE Industry Application Society in 2006 